\title{
El comportamiento de los fenómenos económicos desde una economía positiva
}

\section{The behavior of economic phenomena from a positive economy}

\author{
Freddy Gonzalo Alvarado-Espinoza \\ freddyalvarado@hotmail.com \\ Universidad Agraria del Ecuador \\ Universidad de Guayaquil \\ Ecuador \\ https://orcid.org/0000-0001-7696-7144 \\ Mauro Carlos Tapia Toral \\ mtapia@uagraria.edu.ec \\ Universidad Agraria del Ecuador \\ Ecuador \\ https://orcid.org/0000-0003-3220-4369
}

\section{RESUMEN}

Explicar el comportamiento de los fenómenos económicos desde una economía positiva implica atender atenderla desde el comportamiento de las personas, empresas y gobierno. Bajo esta premisa, en este artículo se evaluaron las distintas posiciones teóricas que sustentan las técnicas de la economía positiva en relación al comportamiento de los fenómenos económicos. Para ello se siguió una metodología de tipo descriptiva y un diseño no-experimental por cuanto no se manipuló deliberadamente la variable economía positiva. Igualmente, la investigación siguió una tipología documental y una postura epistemológica basada en el idealismo-deductivo. Entre sus conclusiones se pudo deducir que el rol que juega en la formulación de las políticas en materia económica, dada la estrecha relación que posee con la economía normativa, permitiendo definir cómo deben ser las políticas públicas, con sus aportes factibles que viabilizan el comportamiento de los mercados.

Descriptores: Fenómenos económicos; Economía positiva; Comportamiento de las personas.

\section{SUMMARY}

Explaining the behavior of economic phenomena from a positive economy implies attending to it from the behavior of people, companies and government. Under this premise, in this article the different theoretical positions that support the techniques of positive economics in relation to the behavior of economic phenomena were evaluated. For this, a descriptive methodology and a non-experimental design were followed 


\section{CIENCIAMATRIA \\ Revista Interdisciplinaria de Humanidades, Educación, Ciencia y Tecnología \\ Año VI. Vol. VI. N¹0. Enero - Julio 2020 \\ Hecho el depósito de ley: pp201602FA4721 \\ ISSN-L: 2542-3029; ISSN: 2610-802X \\ Universidad Nacional Experimental Francisco de Miranda (UNEFM). Santa Ana de Coro. Venezuela}

Freddy Gonzalo Alvarado Espinoza; Mauro Carlos Tapia Toral

because the positive economy variable was not deliberately manipulated. Likewise, the investigation followed a documentary typology and an epistemological stance based on deductive idealism. Among its conclusions, it was possible to deduce that the role it plays in the formulation of economic policies, given the close relationship it has with the normative economy, allowing to define how public policies should be, with its feasible contributions that make possible the behavior of the markets.

Descriptors: Economic phenomena; Positive economy; Behavior of people.

\section{INTRODUCCIÓN}

La economía positiva se limita a la descripción de los fenómenos económicos. El desarrollo de diversas técnicas para analizar mejor o con mayor profundidad estos fenómenos dan lugar a lo que se denomina comportamiento de los fenómenos económicos. Es de indicar, que cuando se hace economía positiva se considera que los economistas actúan como científicos, en las que han de desligarse de la subjetividad para actuar y así poder explicar las relaciones de causa-efecto entre hechos y variables económicas de manera objetiva.

Para Bara (2006), la economía positiva parte de un fenómeno económico y busca encontrar su causa (lo que fue) y sus consecuencias (lo que será). Para ello se trata de establecer una cadena de relaciones causa-efecto entre los distintos fenómenos económicos, de manera que se puedan conocer las consecuencias en la realidad de cualquier cambio en las variables estudiadas. Cabe destacar, que la economía positiva solo puede decir si los medios empleados son correctos para alcanzar un fin determinado. Sin embargo, nada podrá decir sobre si dicho fin es deseable o no. De allí, que desde la economía positiva se pueden explicar la forma en que la sociedad toma sus decisiones de consumo, producción e intercambio de bienes y servicios. Todo ello basándose en la experiencia de la realidad, es decir, en el empirismo, y sin valorar si los fenómenos son deseables o no éticamente, los cuales vienen a definir el comportamiento de los fenómenos económicos. Con base en los argumentos expuestos, y considerando que los fenómenos económicos comprenden todas aquellas actividades sociales realizadas con el fin último de conquistar mayores niveles de bienestar y cuyas actividades sociales, por si mismas definen un espacio de interacción que podemos 


\section{CIENCIAMATRIA \\ Revista Interdisciplinaria de Humanidades, Educación, Ciencia y Tecnología \\ Año VI. Vol. VI. No10. Enero - Julio 2020 \\ Hecho el depósito de ley: pp201602FA4721 \\ ISSN-L: 2542-3029; ISSN: 2610-802X \\ Universidad Nacional Experimental Francisco de Miranda (UNEFM). Santa Ana de Coro. Venezuela \\ Freddy Gonzalo Alvarado Espinoza; Mauro Carlos Tapia Toral}

llamar el sistema económico, en la que se realizan contratos, ofertas, compras y ventas, entre otros, que en conjunto tanto el sistema como las actividades y sus componentes forman parte del fenómeno económico. Con base a esta posición, se propone en este artículo evaluar las posiciones teóricas que sustenta las técnicas de la economía positiva en relación al comportamiento de los fenómenos económicos.

\section{DESARROLLO}

Resulta bastante complicado el encontrar una definición única de la ciencia económica y en consecuencia su objeto de estudio puede aparecer impreciso. Lo afirmado obedece a que tanto los padres de la economía como los economistas de las recientes escuelas de pensamiento no se han puesto de acuerdo sobre la temática debido a los diferentes enfoques teóricos que caracterizan a cada una de las escuelas de pensamiento. Para ello, se presenta la economía positiva que según Fríedman citado por Losa y col (2014), como una ciencia que conoce sistemáticamente y que puede lograr el establecimiento del consenso social sobre las medidas de política económica, con lo que se derivaría una "estabilidad social". Cabe destacar, que el contenido de esta tesis devela que la economía positiva es independiente de cualquier posición ética o juicio valorativo.

\section{Características de la economía positiva}

Dentro de las características fundamentales se pueden mencionar:

- Evade tener juicios de valor económico, ella puede detallar por ejemplo cómo se afecta la inflación si se llega a incrementar la oferta monetaria, pero no da detalles de cuál política económica se debe aplicar.

- Es necesaria para clasificar las políticas económicas y los resultados de aceptabilidad sobre la economía normativa.

- La economía positiva se basa en las causas y efectos de la economía, en cómo se relaciona su comportamiento y cuales hechos se involucran en la evolución y el desarrollo de las teorías económicas.

- Hace estudios sobre los comportamientos económicos. 


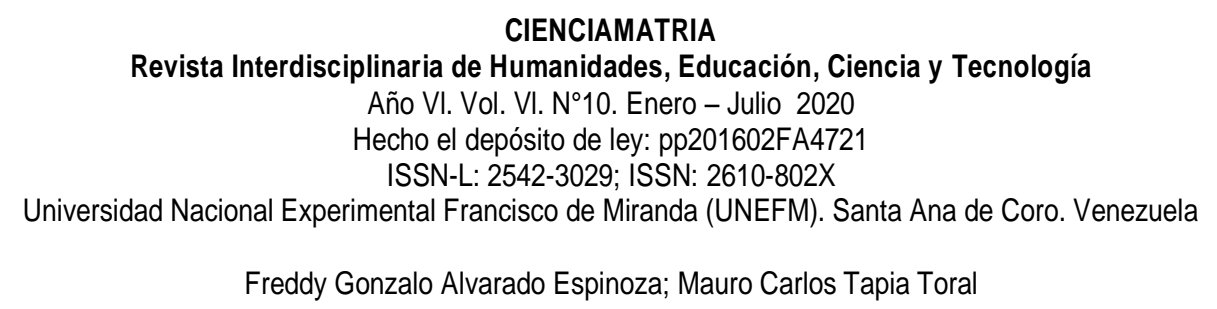

- No da opiniones ya que se centra en declarar y analizar lo que puede ser verificable y comprobable.

- Solo habla de opciones y descripciones

- No establece juicios u opiniones personales.

- No aplica juicios sobre lo que la economía debería estar haciendo, así que establece un nivel de equilibrio sobre precios y cantidades, pero no opina sobre si ese precio es el que debe ser usado para la cantidad.

\section{Importancia de la economía positiva}

Es de indicar que la importancia de la economía positiva se encuentra estrechamente ligada a la economía normativa, dado que ambas son las bases para formular las políticas en materia económica. En este sentido, Ancori y col (2000) dan explicación precisa y clara de cómo deben ser las políticas públicas, pues dado su factibilidad en su análisis y opiniones que dan vida al comportamiento de los mercados. En tanto que al tener una comprensión de cómo funciona la economía positiva se pueden tomar mejores políticas económicas, ya que esta no se basa en los juicios de valor ético o moral como si lo hace la economía normativa.

Es de indicar, que los economistas al momento de emitir sus opiniones muestran las causas y efectos que les permiten tomar las decisiones importantes en esta materia, y es lo que se les llama declaraciones positivas y por tanto deben ser objetivas, de fácil definición y comprobación, pueden ser aceptadas o rechazadas y por tanto corregidas, tomando en cuenta las circunstancias de las evidencias.

\section{Impacto de la economía positiva}

Plantea Black y col (2006), que dicha economía presenta un fuerte impacto en muchos niveles ya que sus estudios y conclusiones, permiten tomar acciones que sirvan para el presente y para el futuro. Así podríamos estar hablando por ejemplo en el caso de un gobierno de cualquier país, que puede tomar decisiones como aumentar el papel moneda basándose en la economía positiva ya que objetivamente pueden tomar una acción haciendo que el consumo aumente y que las tasas de interés en base a créditos bajen. 


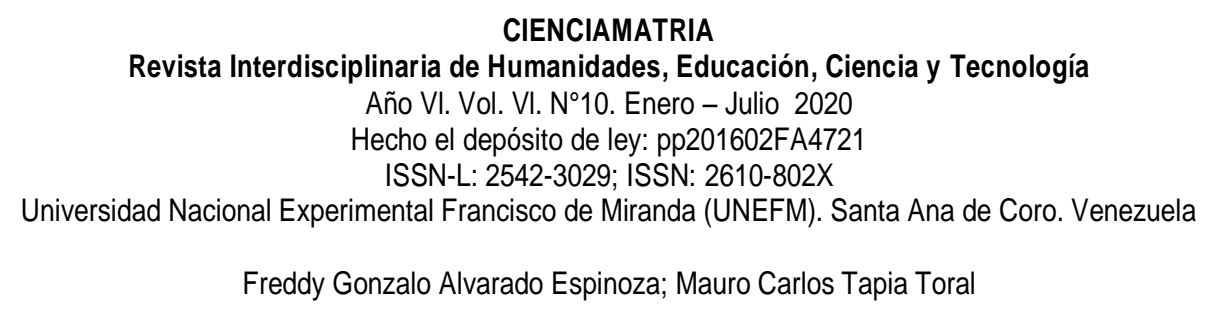

Al mismo tiempo, las empresas pueden asumir deudas en pro de aumentar su capital de trabajo si la economía positiva puede demostrarle que la demanda de sus productos puede aumentar. $Y$ en cuanto a las personas, estas pueden decidir si aumenta o no sus inversiones y ahorros si la economía positiva le pueden dar una explicación objetiva de que su negocio será seguro y estable. De allí que exista la economía como positiva para dar una base real, de tipo científico y precisa a las personas, las empresas y hasta al mismo Gobierno, para tomar decisiones de acuerdo al estudio de las variables económicas.

\section{Economía positiva y negativa}

La llamada economía negativa refiere a la misma economía normativa y cuya diferencia está dada en que la última es subjetiva y la positiva es objetiva, es necesario indicar que ninguna de las dos es mala su diferencia viene dada por la forma en que trabajan, ya que depende de los gobiernos y de la sociedad las reglas y políticas que en materia económica se tomen para satisfacer las necesidades de su población. Ambas están relacionadas ya que tienen su centro en que la economía se lleve de una buena manera, siempre pensando en los factores que la rodean, dando datos y estableciendo conclusiones lógicas que proporcionen beneficios.

\section{Economía positiva vs normativa}

Al respecto Adam Smith, citado por Haakonssen (2006), estableció a la economía como la naturaleza y la causa de la riqueza de las naciones. Por otro lado, David Ricardo la entendía como la distribución de la riqueza entre las diferentes clases sociales, y para Carlos Marx era la dinámica de cómo surgían el desarrollo y la crisis del capitalismo. La diferencia entre ambas se estableció en el siglo XIX a través de la Escuela Clásica con John Stuart Mill y John Neville Keynes. Ellos establecieron que los economistas debían observar un fenómeno de esta índole y luego hacer la hipótesis para explicar el mismo. Posteriormente la hipótesis se llevaría a un hecho real y así establecer el alcance de la teoría postulada. 
Es de indicar que la economía positiva establece descripciones de cómo actúan los fenómenos económicos, de las fuerzas que afectan la misma y trata de dar respuestas de la actividad comercial. Ambas se pueden presentar bajo los siguientes preceptos:

\section{Economía Positiva Vs Normativa}

\section{Cuadro 1}

\begin{tabular}{|l|l|}
\hline Economía positiva & Economía normativa \\
\hline $\begin{array}{l}\text { Establece descripciones de cómo } \\
\text { actúan los fenómenos económicos, de } \\
\text { las fuerzas que afectan la misma y trata } \\
\text { de dar respuestas de la actividad } \\
\text { comercial. }\end{array}$ & $\begin{array}{l}\text { Define criterios que puedan servir de } \\
\text { gúa las decisiones económicas, } \\
\text { debe hacerse. Estas decisiones de } \\
\text { política económica son las que dan las } \\
\text { respuestas a los gobiernos, } \\
\text { estableciendo de manifiesto } \\
\text { componentes subjetivos y juicios de } \\
\text { valor. }\end{array}$ \\
\hline $\begin{array}{l}\text { Se basa en los fenómenos económicos } \\
\text { para responder que debe ser, dicho de } \\
\text { otra manera, sería como se puede } \\
\text { aplicar una acción al mundo real. }\end{array}$ & $\begin{array}{l}\text { Se basa en las preguntas qué es y qué } \\
\text { debe hacerse }\end{array}$ \\
\hline $\begin{array}{l}\text { Es una rama científica objetiva y se } \\
\text { basa en la realidad }\end{array}$ & $\begin{array}{l}\text { Se sujeta a juicios de valor y por tanto } \\
\text { deja de ser científica. }\end{array}$ \\
\hline $\begin{array}{l}\text { Las afirmaciones son testeada, } \\
\text { aprobadas o rechazadas }\end{array}$ & $\begin{array}{l}\text { Se basan en opiniones que no llegan a } \\
\text { ser absolutamente verdaderas o falsas. }\end{array}$ \\
\hline
\end{tabular}

Fuente: elaboración propia

En materia económica es de suma importancia para todo el mundo, no solo a nivel de experiencia sino también a manera de competencia, por lo cual genera fuertes 


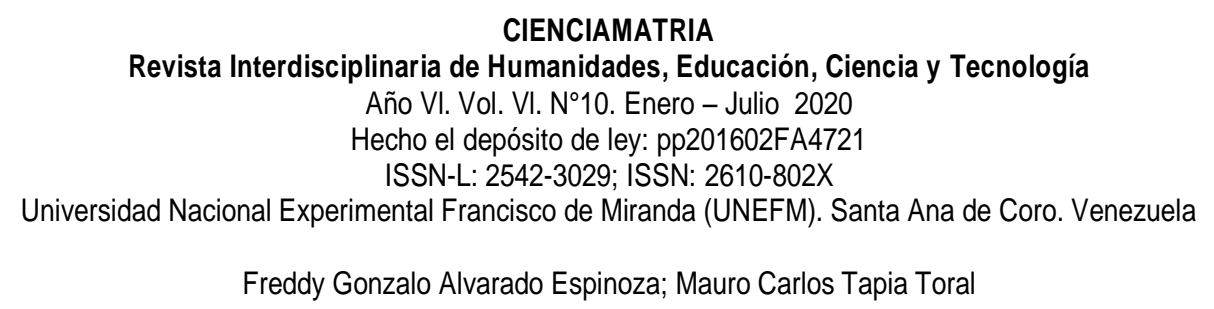

controversias y leyes. Muchos expertos en materia económica establecen que este tema es muy importante y sus opiniones son aceptadas.

\section{Relación entre economía positiva y normativa}

En materia económica es de suma importancia para todo el mundo, no solo a nivel de experiencia sino también a manera de competencia, por lo cual genera fuertes controversias y leyes. Al mismo tiempo, que en esta relación se busca dar explicaciones objetivas del funcionamiento de los fenómenos económicos, se ocupa "de lo que es o podría ser" analizando hechos y datos, y se enmarca en el campo de la teoría económica. Es de destacar que la economía normativa usa como herramientas, las conclusiones de la economía positiva.

Por otra parte, también cabe hacer una distinción entre dos campos de estudio dentro de la economía:

- La economía positiva busca hacer una descripción de los fenómenos económicos, en ella se evalúan las fuerzas que afectan a la economía e intenta predecir las consecuencias de su actividad.

- La economía normativa define algunos criterios que guíen las decisiones económicas. Responde a la pregunta de "qué debe ser", "qué debe hacerse".

\section{METODOLOGÍA}

La presente investigación está delimitada dentro del área de la economía, específicamente en referencia a la economía positiva. La naturaleza de la investigación es de tipo descriptiva, porque se propone caracterizar la presencia de la economía positiva en el comportamiento de los fenómenos económicos. El diseño fue noexperimental por cuanto no se manipuló deliberadamente la variable economía positiva. Igualmente, la investigación siguió una tipología documental, en la que según Balestrini (2012), la recolección de la información se encuentra vinculada a la delimitación de un diseño bibliográfico a partir de la consulta de libros y documentos, los cuales sirvieron de 


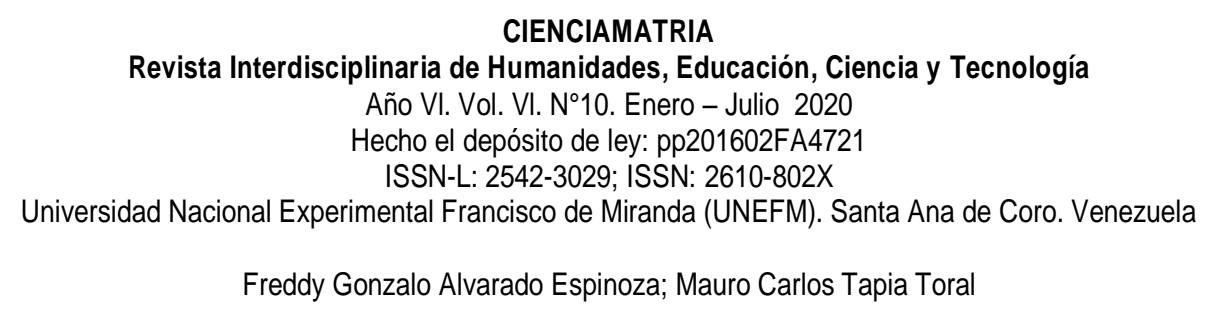

apoyo y respaldo al estudio planteado. De acuerdo con su intención, la misma asumió una postura epistemológica basada en el idealismo-deductivo, en tanto que se partió del conocimiento previo de las teorías económicas para presentar conclusiones hermenéuticas de carácter objetivo acerca de sus teorías.

\section{CONCLUSIONES}

Posterior al análisis de las teorías que apoyan y sustentan la tesis de una economía positiva en el marco del comportamiento de los fenómenos económicos fue posible develar:

- La economía positiva es una ciencia de tipo objetiva y por eso usa la base del método científico como cualquiera de las otras ciencias. El hecho de que la economía tenga una posición en las relaciones de los seres humanos y que el economista haga sus investigaciones plantea que pueden existir dificultades para alcanzar un nivel de objetividad.

- Entre las características que distinguen a la economía positiva, es propicio develar cómo puede afectar la inflación, en caso que en las regiones se dé un incremento de la oferta monetaria, ello ayudaría a normatizar las políticas económicas requeridas para atacarla, entendiendo que ella se basa en las causas y efectos y en la relación de su comportamiento para el desarrollo de las teorías económicas.

- Su importancia viene a estar manifiesta por el rol que juega en la formulación de las políticas en materia económica, dada la estrecha relación que posee con la economía normativa, permitiendo definir cómo deben ser las políticas públicas, con sus aportes factibles que viabilizan el comportamiento de los mercados.

- El impacto de la Economía Positiva puede ser medido a través de su intervención para lograr definir las acciones pertinentes en economía de modo presente y futuro para decisiones gubernamentales, a nivel empresarial y hasta personal, por estar fundamentada en una base real, científico y precisa tanto para las personas, las empresas como hasta al mismo Gobierno. 


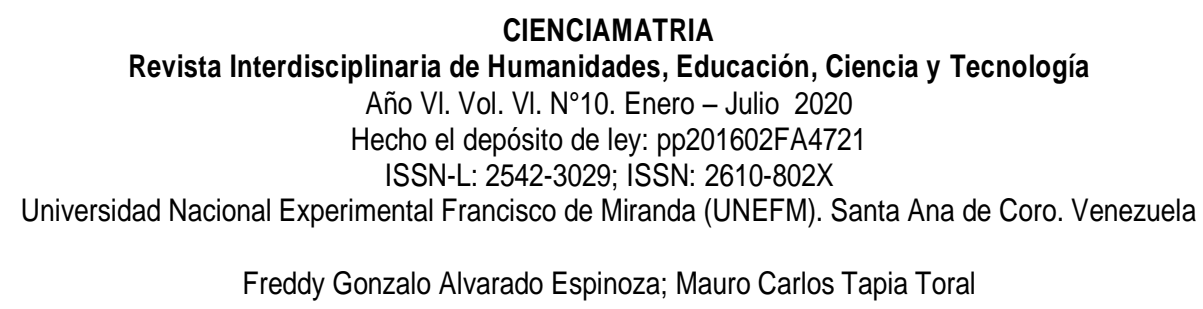

- La relación de la economía positiva con la normativa, está caracterizada en ser elementos de complementariedad, en tanto que la primera hace la descripción de lo que sucede en la economía y este análisis es la respuesta para desarrollar otras formas de análisis con una mejor o mayor profundización del fenómeno que estudia y la economía normativa establece opiniones sobre lo que se debe alcanzar en las actividades económicas.

\section{REFERENCIAS CONSULTADAS}

1. Ancori, B., Bureth, A. y Cohendet, P. (2000). "The Economics of Knowledge: The Debate about Codification and Tacit Knowledge", Industrial and Corporate Change, Vol. 9, № 2, junio 2000.

2. Balestrini, M. (2012). Cómo se elabora el proyecto de investigación. Caracas, Consultores Asociados

3. Black, R. A. "What did Adam Smith Say About Self-Love?" Journal of Markets \& Morality 9.1. (2006): 7-34.

4. Bara, R. (2006): Finanzas Públicas y Decisiones Públicas: Un Enfoque de Economía Política, Consejo Profesional de Ciencias Económicas de la Ciudad Autónoma de Buenos Aires, $1^{\text {a }}$ edición.

5. Haakonssen, Knud (2006). The Cambridge Companion to Adam Smith, Cambridge University Press. ISBN 978-0-521-77924-1

6. Losa Carmona, A y Beltrán Abellán, J (2014). Mercados Laborales Locales y Riesgo de Exclusión en la Región de Murcia, Ediciones de la Universidad de Murcia, Murcia.

7. Salinas Jiménez, J. y Delgado Rivero, F. (2012): Impuestos, crecimiento económico y bienestar: una visión panorámica, Documento presentado en el XXIV Seminario Regional de Política Fiscal, Santiago de Chile, 24-26 de enero.

\section{REFERENCES CONSULTED}

1. Ancori, B., Bureth, A. and Cohendet, P. (2000). "The Economics of Knowledge: The Debate about Codification and Tacit Knowledge," Industrial and Corporate Change, Vol. 9, No. 2, June 2000. 
2. Balestrini, M. (2012). How the research project is made. Caracas, Associated Consultants

3. Black, R. A. "What did Adam Smith Say About Self-Love?" Journal of Markets \& Morality 9.1. (2006): 7-34.

4. Bara, R. (2006): Public Finance and Public Decisions: An Approach to Political Economy, Professional Council of Economic Sciences of the Autonomous City of Buenos Aires, 1st edition.

5. Haakonssen, Knud (2006). The Cambridge Companion to Adam Smith, Cambridge University Press. ISBN 978-0-521-77924-1

6. Losa Carmona, A and Beltrán Abellán, J (2014). Local Labor Markets and Exclusion Risk in the Region of Murcia, Editions of the University of Murcia, Murcia.

7. Salinas Jiménez, J. and Delgado Rivero, F. (2012): Taxes, economic growth and well-being: an overview, Document presented at the XXIV Regional Seminar on Fiscal Policy, Santiago, Chile, January 24-26. 